\title{
Czech Participation in the INTEGRAL Satellite: A Review
}

\author{
R. Hudec, M. Blažek, V. Hudcová
}

\begin{abstract}
The ESA INTEGRAL satellite, launched in October 2002, is the first astrophysical satellite of the European Space Agency ESA with Czech participation. The results of the first 7 years of investigations of various scientific targets e.g. cataclysmic variables, blazars, X-ray sources, and GRBs with the ESA INTEGRAL satellite with Czech participation are briefly presented and discussed.
\end{abstract}

Keywords: high-energy astrophysics, high-energy satellites.

\section{Introduction}

The ESA INTEGRAL project was the first ESA project in space astronomy with official Czech participation based on a collaboration agreement between the ESA and the Czech Republic, i.e. prior to full membership of the Czech Republic in ESA. INTEGRAL (International Gamma-Ray Astrophysics Laboratory) satellite has now been more than 7 years in orbit, so some general conclusions may be drawn at this point. There are four co-aligned instruments on board INTEGRAL: (1) IBIS gamma-ray imager (15 keV-10 MeV, full coded field of view (FOV) $8.3 \times 8$ deg, 12 arc min FWHM), (2) SPI gamma-ray spectrometer $(12 \mathrm{keV}-8 \mathrm{MeV}$, full coded FOV $16 \times$ $16 \mathrm{deg}),(3)$ JEM-X X-ray monitor (3-35 keV, fully illuminated FOV diameter $4.8 \mathrm{deg}$ ), and (4) OMC optical monitoring camera (Johnson $V$ filter, FOV $5 \times 5 \mathrm{deg}$ ) (Winkler et al. 2003). These experiments allow simultaneous observation in the optical, medium X-ray, hard X-ray, and gamma spectral region (or at least a suitable upper limit) for each object, assuming that it is inside the field of view. The basic codes of the observations are as follows: (a) Regular (weekly) Galactic Plane Scans (GPS) $\left(-14 \mathrm{deg}<b_{\mathrm{II}}<+14 \mathrm{deg}\right.$ ), (b) Pointed observations (AO), (c) Targets of opportunity ( $\mathrm{ToO}$ ). In this paper we deal with examples of observations and analyses of INTEGRAL data with our participation, focusing on two categories of objects, namely cataclysmic variables (CVs) and blazars.

\section{Czech involvement in the INTEGRAL project}

Czech involvement in the ESA INTEGRAL project started in 1996 by inviting the first author of this paper to the OMC and ISDC consortia, based on a collaboration agreement between ESA and the Czech
Republic. Then our participation focused on ISDC and OMC.

In OMC (Optical Monitoring Camera), the participation focused on various software packages such as OMC PS (OMC Pointing Software) for Integral ISOC, and also on the design, development and operation of OMC TD (Test Device), a ground-based camera with output analogous (pixel size 18 arcsec) to the real OMC. For ISDC (Integral Science and Data Center), located in Versoix, Switzerland, the main part of the contribution focused on providing manpower, i.e. one person working within the team, with various responsibilities and involvements in the ISDC operations. As for the scientific responsibilities, the first author of this paper was delegated to lead the study of cataclysmic variables, and he also became a member of the working groups on gammaray bursts (GRBs) and AGNs. In this paper we very briefly summarize the scientific achievements obtained in these directions. In addition, we participated in developing and operating the dedicated robotic telescopes, considered as the ground-based segment of the project, and in delivering supplementary optical data for satellite triggers. These efforts were solved mainly by young research fellows and by students

The Czech scientific participation focused on topics allocated by the INTEGRAL bodies, mostly cataclysmic variables but also blazars and some other objects such as Gamma-Ray Bursts (GRBs).

\section{Integral and cataclysmic variables}

The field of cataclysmic variables (CVs) and related objects was delegated to the responsibility of the first author of this paper. The results of hard X-ray detections of these binary galactic objects are surprising. The soft X-ray emission of the group was already 


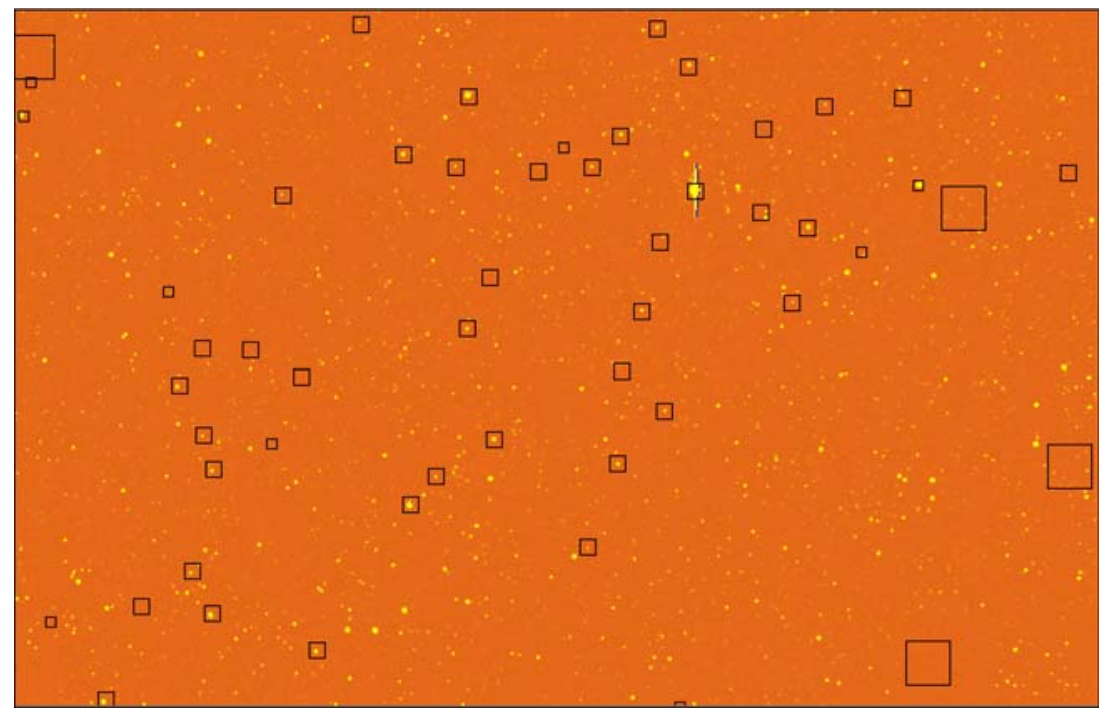

Fig. 1: Sub-windowing of an OMC CCD frame by OMC PS (Pointing Software). The sub-windows generated cover images of astrometric and photometric stars, and also the positions of various objects of interest from catalogues. Up to 100 sub-windows can be created per frame

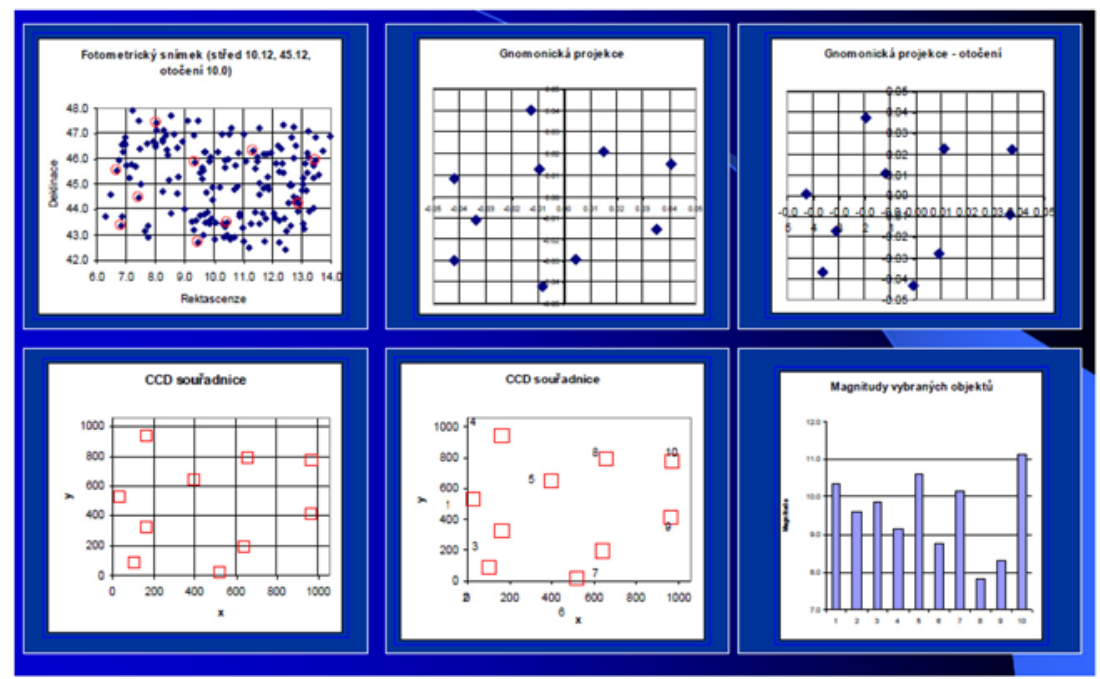

Fig. 2: Selection of objects by OMC photometric shots by OMC PS (Pointing Software)
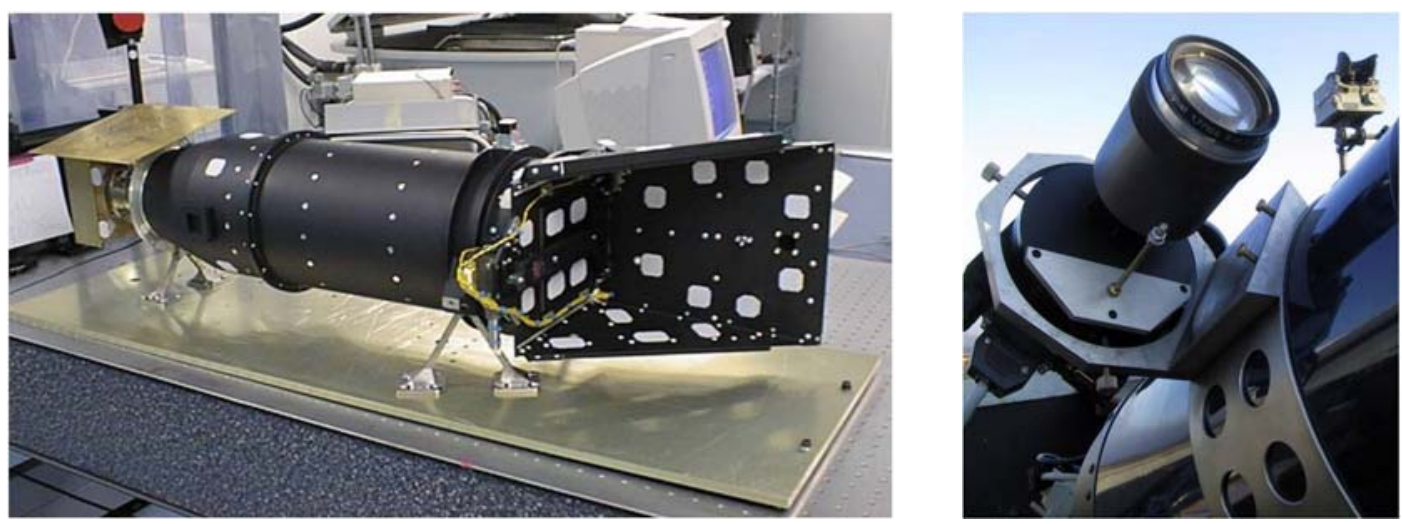

Fig. 3: The OMC on board camera Right: The OMC Test Device operated in Ondřejov prior to the launch of INTEGRAL 


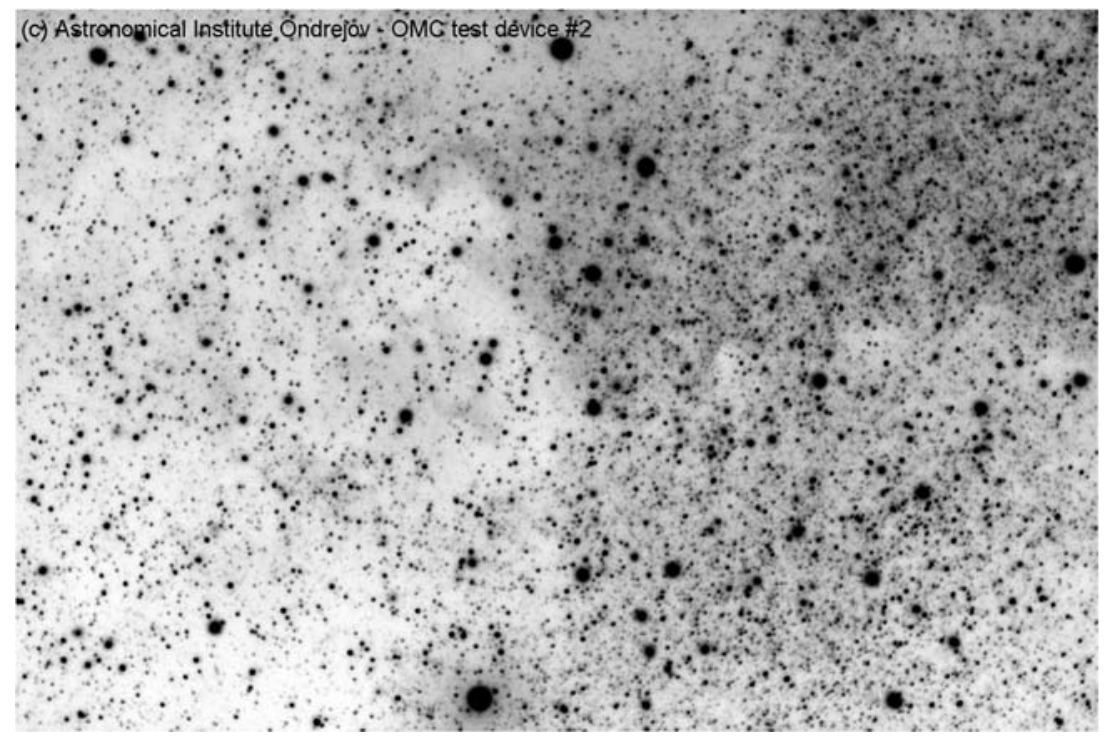

Fig. 4: Image from the OMC Test Device operated in Ondřejov prior to the launch of INTEGRAL. The pixel size of the CCD camera was identical to those of real OMC (17 arcsec)

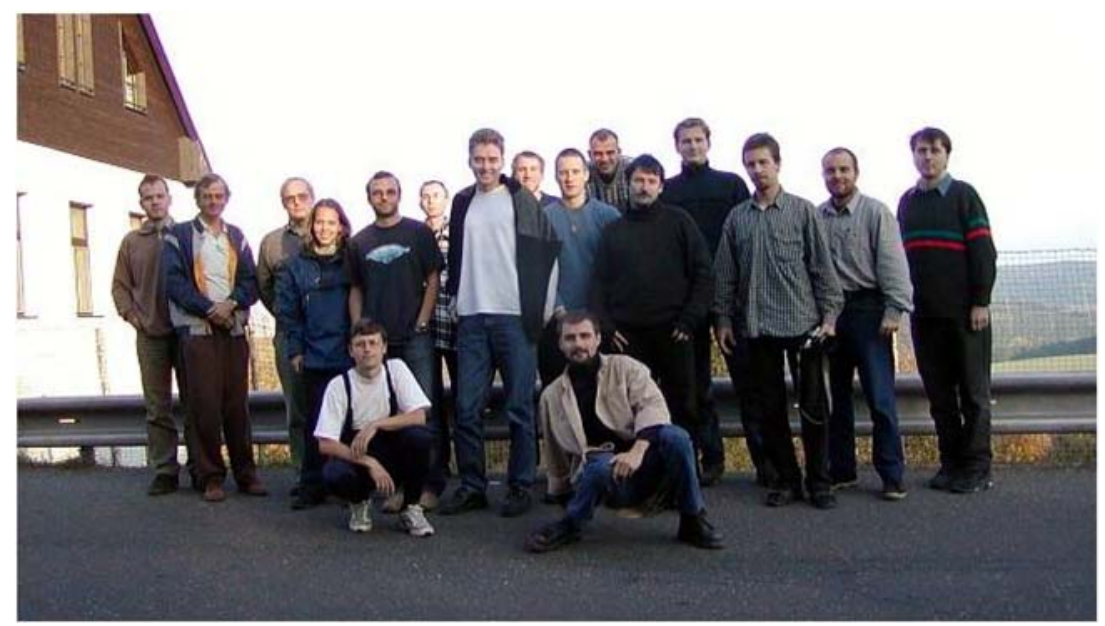

Fig. 5: The Czech INTEGRAL team at the 2nd IBWS INTEGRAL/BART workshop held in Kostelni Strimelice in October 2003

known in advance, but the hard X-ray extension to (in some cases) more than $80 \mathrm{keV}$ was a new discovery. These findings even led to considerations that CVs may represent a contribution to the galactic $\mathrm{X}$ ray background.

So far, 32 cataclysmic variables $(\mathrm{CVs})$ have been detected by the INTEGRAL IBIS gamma-ray telescope (surprisingly, this is more than had been expected before launch, and represents almost 10 percent of the INTEGRAL detections). $22 \mathrm{CVs}$ were seen by IBIS and found by the IBIS survey team (Barlow et al. 2006, Bird et al. 2007, Galis 2008), based on the correlation of the IBIS data and the Downes CV catalogue (Downes et al. 2001). Four sources are CV candidates revealed by optical spectroscopy of IGR sources (Masetti et al. 2006), i.e. new CVs, not in the Downes catalogue. They are mainly magnetic systems: 22 are confirmed or probable IPs, 4 probable magnetic CVs, 3 polars, 2 dwarf novae, 1 unknown. The vast majority have an orbital period $P_{\text {orb }}>3 \mathrm{hr}$, i.e. above the period gap (only one has $P_{\text {orb }}<3 \mathrm{hr}$ ), 5 objects are long-period systems with $P_{\text {orb }}>7 \mathrm{hr}$.

The long life time of the satellite allows longterm variability studies, albeit limited by observation sampling. At least in some cases, the hard X-ray fluxes of CVs seen by INTEGRAL exhibit time variations, very probably related to activity/inactivity states of the objects. The spectra of CVs observed by IBIS are similar in most cases. The power law or thermal bremsstrahlung model compares well with the previous high-energy spectral fits (de Martino et al. 2004, Suleimanov et al. 2005, Barlow et al. 2006). 

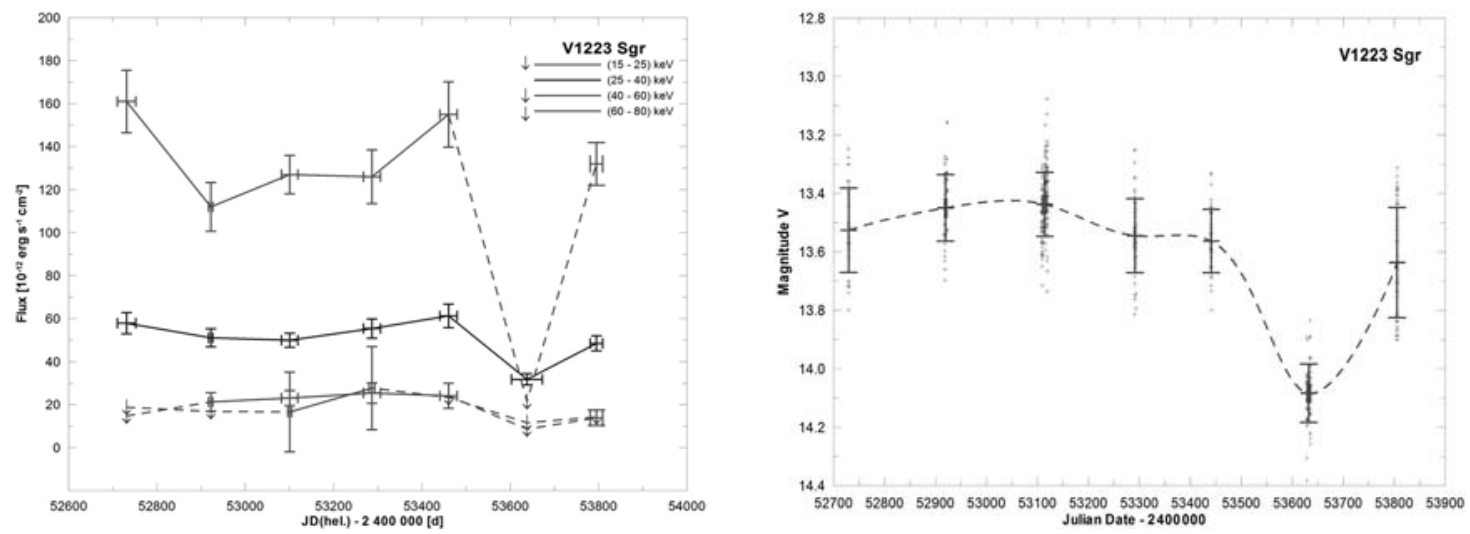

Fig. 6: The IBIS gamma-ray light curve for CV V1223 Sgr. The fluxes especially in the (15-25) keV and (25-40) keV bands are long-term variables with a significant drop around MJD 53 650. Optical variations are correlated with the changes in the (15-25) keV, (25-40) keV and (40-60) keV spectral bands with correlation coefficient $0.81,0.82$ and 0.89 , respectively. The fluxes from INTEGRAL/JEM-X were persistent within their errors in the monitored time period. Right: The OMC optical (V band) light curve for V1223 Sgr

Another surprise is the fact that while the group of IPs represents only $\sim 2$ percent of the catalogued $\mathrm{CVs}$, it nevertheless dominates the group of CVs detected by IBIS. More such detections and new identifications can therefore be expected, as confirmed by our search for IPs in the IBIS data, which provided 6 new detections (Galis et al. 2008). Many CVs covered by the Core Program (CP) remain unobservable by IBIS because of the short exposure time, but new CVs have been discovered. IBIS tends to detect IPs and asynchronous polars: in hard X-rays, these objects seem to be more luminous (up to a factor of 10) than synchronous polars. Detection of CVs by IBIS typically requires $150-250 \mathrm{ksec}$ of exposure time or more, but some of them remained invisible even after $500 \mathrm{ksec}$. This can however, at least in some cases, be related to the activity state of the sources — the hard X-ray activity is temporary or variable.

For short-term variations, there is an indication for a hard X-ray flare in a CV system, namely V1223 Sgr, seen by IBIS (a flare lasting for $\sim 3.5 \mathrm{hr}$ during revolution 61 (MJD 52743), with the peak flux $\sim 3$ times the average (Barlow et al. 2006)) These flares were seen in the optical already in the past by a ground-based instrument (duration of several hours) (van Amerongen \& van Paradijs 1989). This confirms the importance of the OMC-like instrument (preferably with the same FOV as a gamma-ray telescope) on board gamma-ray satellites: even with the $V$ limiting mag 15 , it can provide valuable optical simultaneous data to the gamma-ray observations. Analogous flares are also known for other IPs in the optical, but not in hard X-rays. An example is TV Col (Hudec et al. 2005), where 12 optical flares have been observed so far, five of them on archival plates from the Bamberg Observatory, and remainig ones by others observers. TV Col is an IP and the optical counterpart of the X-ray source 2A0526-328 (Cooke et al. 1978). This is the first CV discovered through its X-ray emission, newly confirmed as an INTEGRAL source. The physics behind the outbursts in IPs is either disk instability or an increase in the mass transfer from the secondary.

\section{INTEGRAL and blazars}

Blazars are among the most important and also most optically violently variable extragalactic high-energy objects. Below we list a few examples of blazars analyzed with INTEGRAL observations. We focus on objects found by data mining in the INTEGRAL archive for faint and hidden objects. For more details on blazar analyses with INTEGRAL, see Hudec et al. (2007). In addition, successful blazar observations were performed mostly in the ToO regime.

\begin{tabular}{|c|c|c|c|c|c|c|}
\hline source & FA & DEC & \multicolumn{2}{|c|}{ Gal coord } & $z$ & type \\
\hline ES $0647+250$ & $\infty 65046.6$ & +250300 & 190.283 & 10.896 & 0.2030 & BL Lac \\
\hline PKS 0823-223 & 082801.5729 & .223027 .204 & 243.990 & 8930 & 09100 & possible Q \\
\hline IES 2344+514 & 234704.919 & +514217.87 & 112.892 & -9.903 & 0.0440 & BL Lac \\
\hline BC $0149+710$ & 015325.8511 & +711506.463 & 127.920 & 8.983 & 0.0220 & 0 \\
\hline $4 C 47.08$ & 030335.2422 & +471616.276 & 144.986 & -9.863 & 0.4750 & BL Lac \\
\hline $87 G B$ 02109+5130 & 010328.1 & +432259 & & & & BL Lac \\
\hline BL Lac & 220243.2914 & +421639.980 & 92.590 & -10.441 & 0.0688 & BL Lac \\
\hline S5 $0716+714$ & 072153.4485 & +712036.363 & 143.981 & 28018 & 0,3000 & BL Lac \\
\hline S5 $0036+710$ & OS 4124.3653 & +705342.173 & 143.541 & 34.426 & 2.1720 & a \\
\hline 3C 454.3 & 225357.7479 & +160853561 & 86.111 & -38.184 & 0.8590 & $a$ \\
\hline $3 C 279$ & 125611.1665 & .054721 .525 & 305.104 & 57.062 & 0.5362 & a \\
\hline 36273 & 22306.6997 & .020303598 & -11.010 & 4.380 & 0.1583 & a \\
\hline PKS1830-211 & 183339.888 & -210339.77 & 12.166 & -5.712 & 2.5070 & 0 \\
\hline Mrk421 & 110427.3139 & +381231.799 & 179.832 & 65.032 & 0.0300 & BLLac \\
\hline J1656.3-3302 & 165619.2 & -330148 & 350.504 & 6.361 & & ? \\
\hline IGR J22517*2218 & 225153.498 & +221737.29 & 89.620 & -32.750 & 3.6680 & Q \\
\hline PKS0537-441 & 053843.5 & -440505 & 250.078 & .31 .110 & 0.8960 & BL Lac \\
\hline $3 \mathrm{C} 66 \mathrm{~A}$ & 022239.6115 & .43 $02 \quad 07.799$ & 140.143 & $\cdot 16.767$ & 0.4440 & Blazar \\
\hline Mrk 501 & 1653522167 & +394536609 & 63600 & 38.859 & 0.0336 & B. Lac \\
\hline 1ES2 & 234704.919 & +514217.87 & 112.892 & -9.908 & 0.0440 & BLLac \\
\hline 1ES $1959+650$ & 195959.8521 & 550854653 & 98003 & 17.670 & 0.0420 & Blazzar \\
\hline
\end{tabular}

Fig. 7: The list of blazars observed by the INTEGRAL satellite in hard X-rays (IBIS ISGRI) 

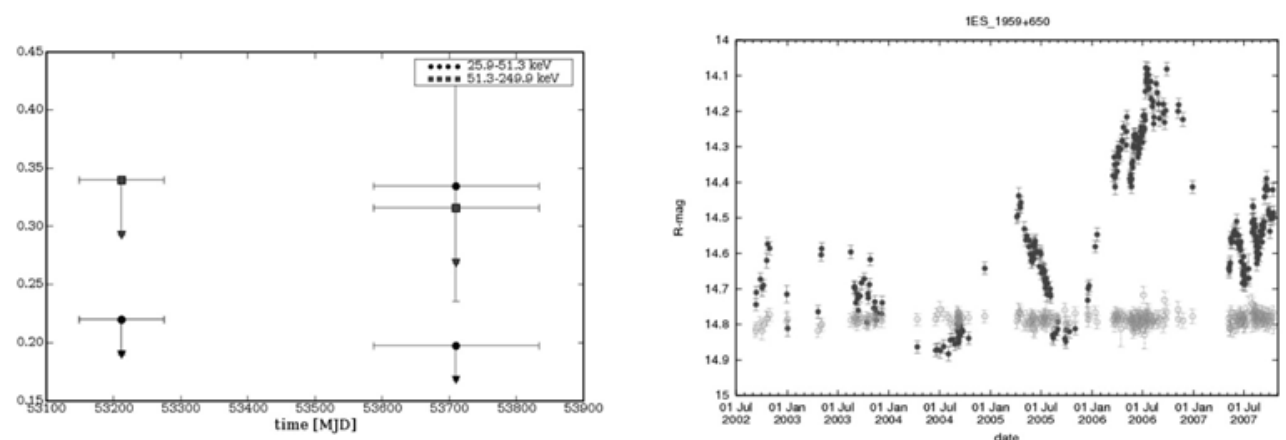

Fig. 8: Left: IBIS gamma-ray light curve of 1ES 1959+650. This blazar is visible in the INTEGRAL IBIS gamma-ray imager only in the data set corresponding to the optical flare. Right: Optical light curve of blazar 1ES 1959+650 (Tuorla Observatory blazar monitoring program). In hard X-rays (IBIS), the blazar is visible only during the large optical flare in the 2nd half of 2006
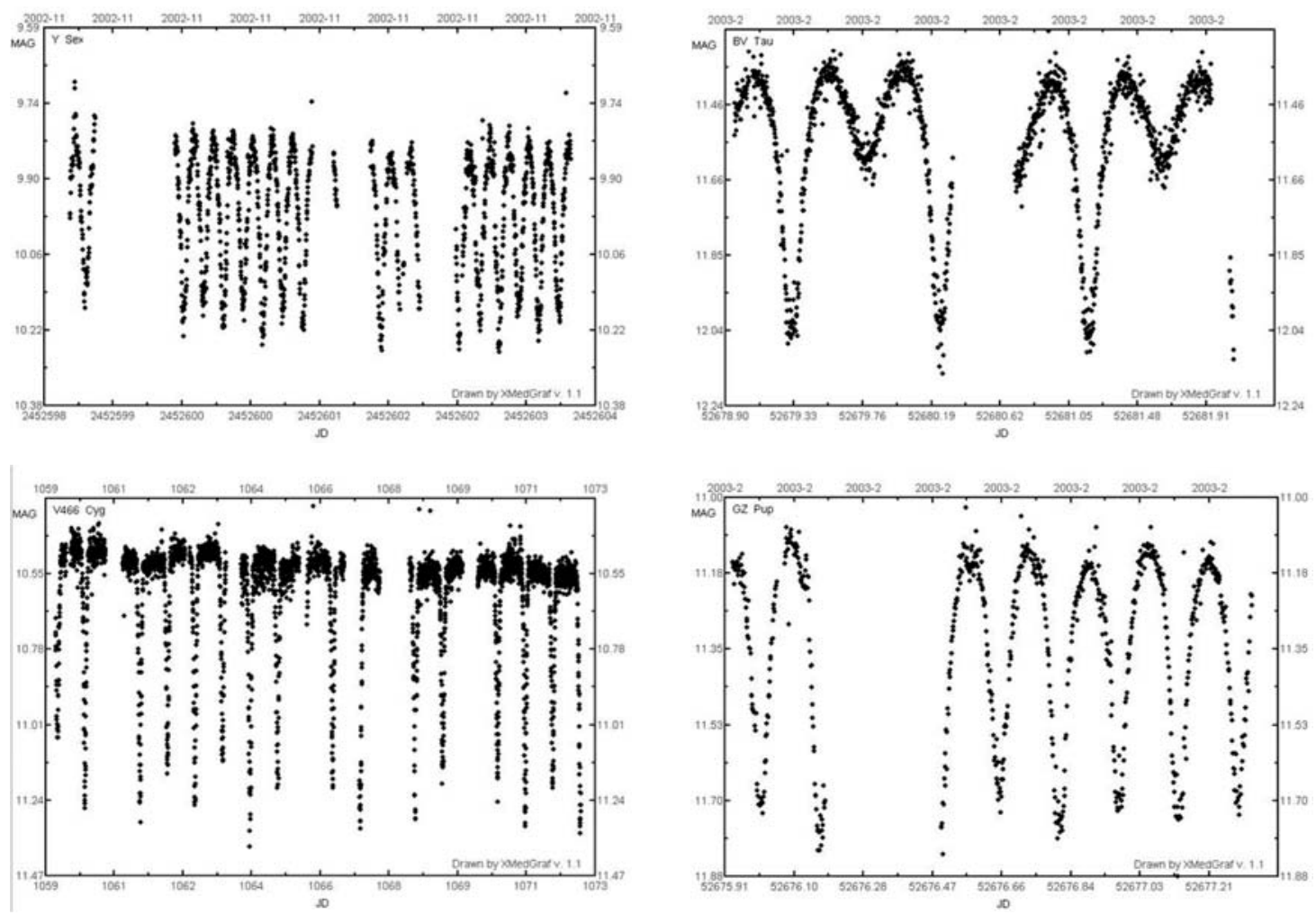

Fig. 9: The light curves of selected eclipsing binaries obtained by the OMC camera

The large collaboration led by E. Pian can serve as an example (Pian et al. 2007). We have developed procedures to access faint blazars in the IBIS database. Blazar 1ES 1959+650 can serve as an example. This blazar is a gamma-ray loud variable object visible by IBIS in 2006 only, invisible in total mosaics and/or other periods. The optical light curve available for this blazar confirms the relation of active gamma-ray and active optical state.

\section{OMC optical camera}

The small optical camera on board INTEGRAL satellite OMC delivered a great deal of valuable si- multaneous optical data for observations of gammaray burst sources. However this is the case only for some triggers, as the field of view (FOV) of OMC is much smaller than the FOV of the mainly used instrument on INTEGRAL, namely IBIS (5 vs. 8 degrees). On the other hand, OMC proved to be an efficient tool for optical objects without gamma-ray counterparts, such as eclipsing binaries. For these objects, the uninterrupted nature (no day/night cycles) of space-based observations was positive for studying the light curves and for determining the times of the minima. 


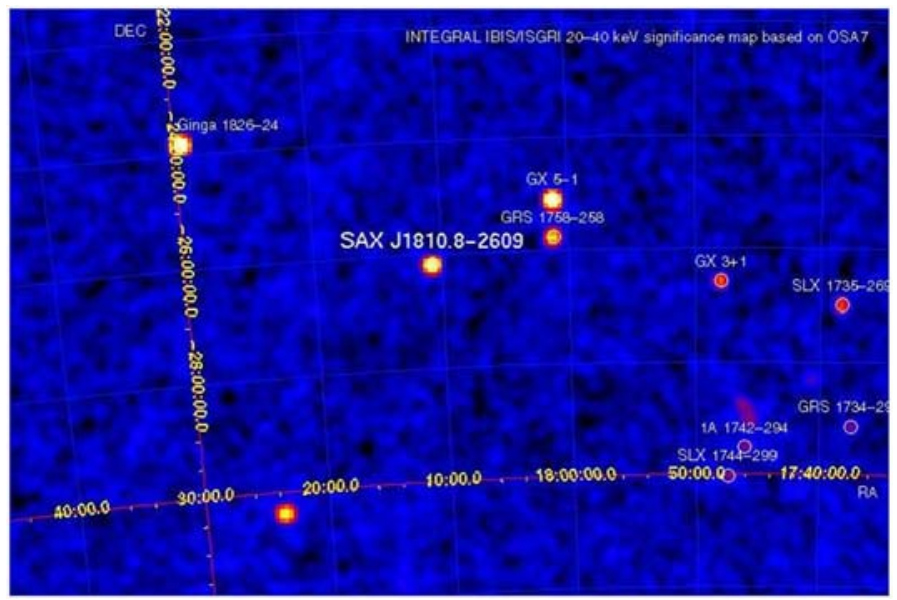

Fig. 10: We observed the longest and brightest outburst of the (most likely) neutron-star low-mass X-ray binary SAX J1810.8-2609 observed by INTEGRAL so far. During the observation period spanning from Sept. 12 to Sept. 21, 2007, the binary system SAX J1810.8-2609 became increasingly brighter. It even emitted a type I X-ray burst, whose flux exceeded that of the Crab nebula (IBIS image)
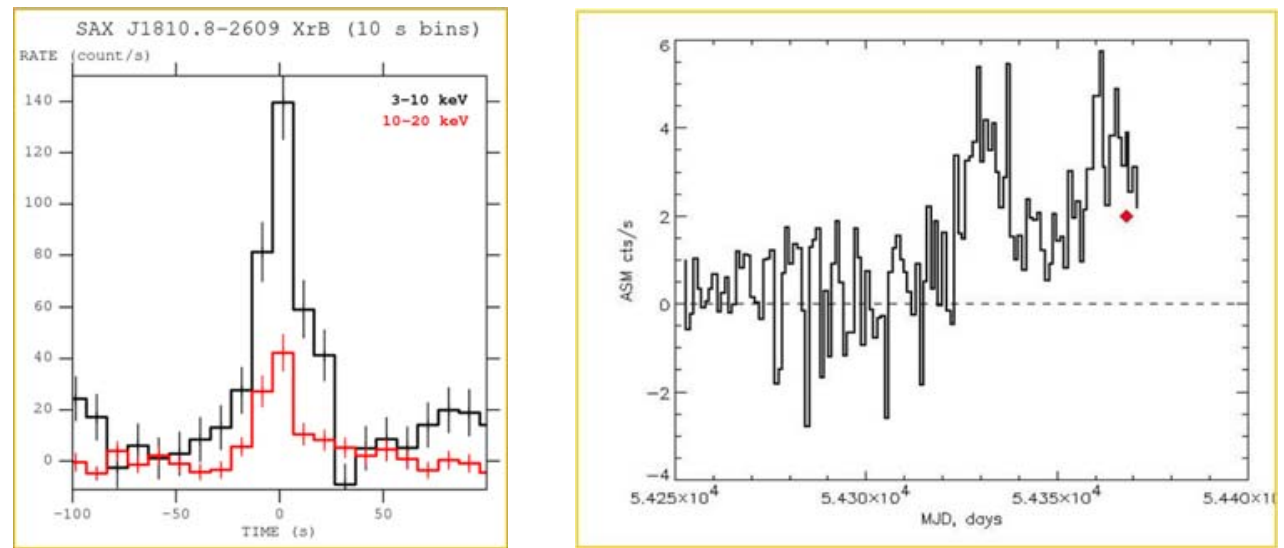

Fig. 11: Left: The light curve of the type I X-ray burst observed by INTEGRAL/JEM-X. The time of the peak corresponds to 2007-09-24T19:53:06, when SAX J1810.8-2609 reached a flux of $1.3 \pm 0.2$ Crab (3-10 keV; shown in black - upper light curve) and 1.1 $\pm 0.3 \mathrm{Crab}$ (10-20 keV; shown in red - bottom light curve). Right: RXTE Light Curve of the object with the INTEGRAL observation indicated by a dot
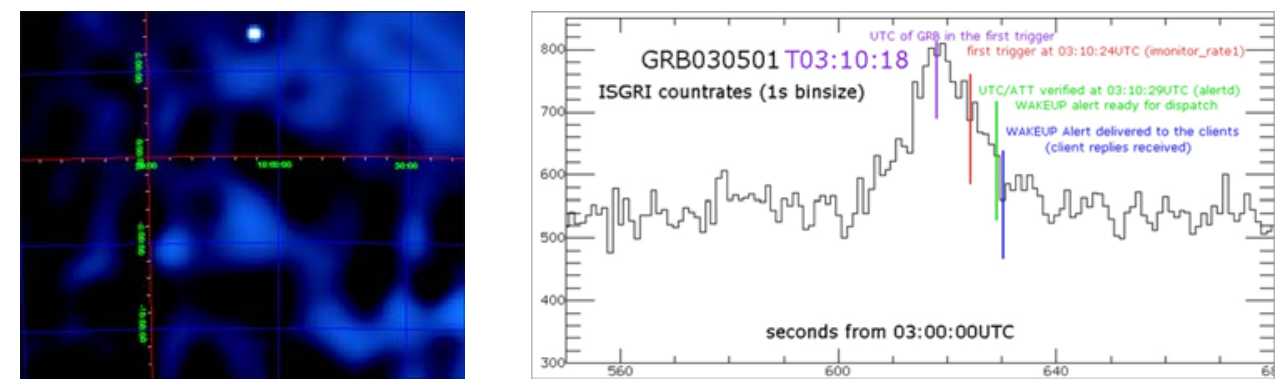

Fig. 12: "Labor day" GRB030501 allocated to our responsibility. Left: The GRB image by SPI, right: the obtained light curve

\section{GRBs and other objects}

Additional results have also been obtained with our participation for other types of high-energy sources, the two most important results being briefly mentioned below. These results are illustrated in Figures 11 and 12, one for neutron star low-mass X-ray binary, the other for a gamma-ray burst (GRB). 


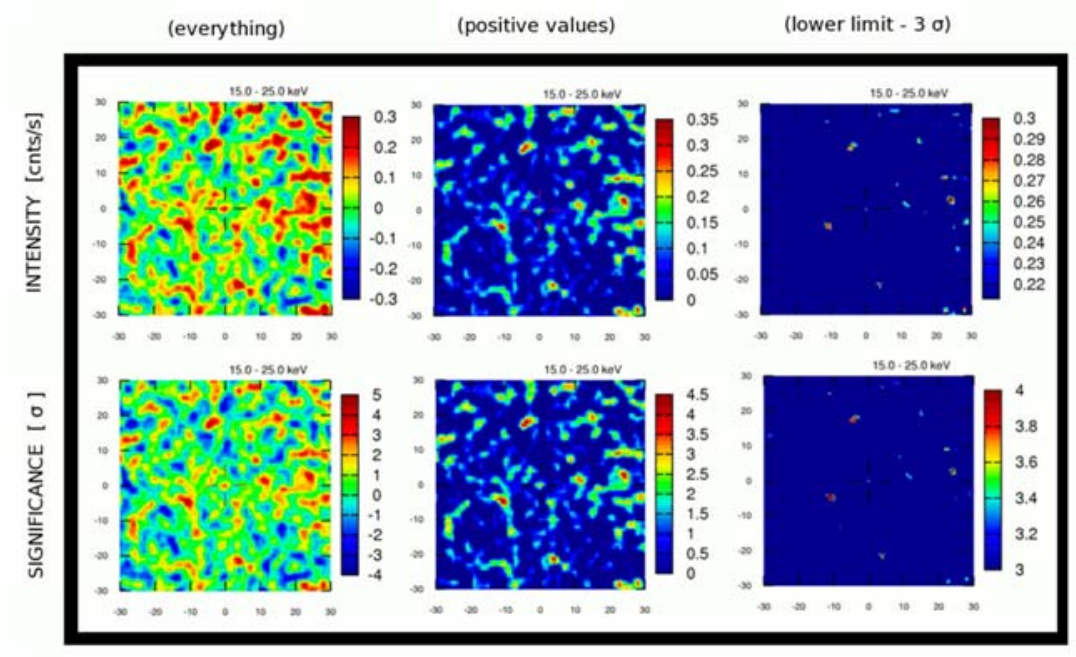

Fig. 13: An example of a study for faint sources in IBIS data and noise reduction - comparison of visualization methods. $\mathrm{XY}$ Ari is an example of a newly detected CV (IP) in IBIS data

\section{Conclusion}

In is obvious that the INTEGRAL satellite is an effective tool for analyzing CVs and blazars. So far, 21 blazars, $32 \mathrm{CVs}$ and 3 symbiotics have been detected, with the number increasing with time. The successful observations of CVs by INTEGRAL provide proof that CVs can be successfully detected and observed in hard X-rays with INTEGRAL (for most CVs these are considerably harder passbands than were possible previously). These results show that more CVs (and in harder passbands) will be detectable with increasing integration time. There is also an increasing probability of detecting objects in outbursts, high and low states, etc. Simultaneous hard X-ray and optical monitoring of CVs and blazars (or at least suitable upper limits) can provide valuable inputs for a better understanding of the physical processes that are involved.

\section{Acknowledgement}

We ackowledge ESA PECS project C98023, grants 205/08/1207 and 102/09/0997 provided by the Grant Agency of the Czech Republic and MSMT KONTAKT Project ME09027.

\section{References}

[1] Barlow, E. J., Knigge, C., Bird, A. J., et al.: MNRAS, 2006, 372, 224.

[2] Bianchini, A., Sabbadin, F.: IBVS, 1985, 2 751, 1.

[3] Bird, A. J., Malizia, A., Bazzano, A., et al.: ApJ Suppl. S., 2007, 170, 175.
[4] Cooke, B. A., Ricketts, M. J., Maccacaro, T., et al.: $M N R A S, 1978, \mathbf{1 8 2}, 489$.

[5] de Martino, D., Matt, G., Belloni, T., et al.: $A \&$ A, 2004, 415, 1009 .

[6] Downes, R. A., Webbink, R. F., Shara, M. M., et al.: PASP, 2001, 113, 764.

[7] Garnavich, P., Szkody, P.: PASP, 1988, 100, 1522.

[8] Hudec, R.: BAIC, 1981, 32, 93.

[9] Hudec, R., Šimon, V., Skalický, J.: The Astrophysics of Cataclysmic Variables and Related Objects, Proc. of ASP Conf., 2005, Vol. 330. San Francisco : ASP, p. 405.

[10] Hudec, R., Šimon, V., Munz, F., Galis, R., Strobl, J.: INTEGRAL results on cataclysmic variables and related objects, presented at INTEGRAL Science Workshop, Sardinia, Oct 2007, http://projects.iasf-roma.inaf.it/integral/ Integral5thAnniversaryPresentations.asp.

[11] Ishida, M., Sakao, T., Makishima, K., et al.: MNRAS, 1992, 254, 647.

[12] King, A. R., Ricketts, M. J., Warwick, R. S.: MNRAS, 1979, 187, 77.

[13] Masetti, N., Morelli, L., Palazzi, E., et al.: $A \&$ A, 2006, 459, 21.

[14] Meintjes, P. J., Raubenheimer, B. C., de Jager, O. C., et al.: ApJ, 1992, 401, 325. 
[15] Motch, C., Haberl, F.: Proceedings of the Cape Workshop on Magnetic Cataclysmic Variables, San Francisco : ASP, 1995, Vol. 85, p. 109.

[16] Motch, et al.: $A \& \&$ A, 1996, 307, 459.

[17] Mürset, U., Jordan, S., Wolff, B.: Supersoft XRay Sources, LNP, Vol. 472, p. 251.

[18] Patterson, J., Skillman, D. R., Thorstensen, J., et al.: PASP, 1995, 107, 307.

[19] Šimon, V., Hudec, R., Štrobl, J., et al.: The Astrophysics of Cataclysmic Variables and Related Objects, Proc. of ASP Conf. 2005, Vol. 330. San Francisco : ASP, p. 477.

[20] Sokoloski, J. L., Luna, G. J. M., Bird, A. J., et al.: $A A S, 2005, \mathbf{2 0 7}, 3207$.

[21] Suleimanov, V., Revnivtsev, M., Ritter, H., $A \&$ A, 2005, 443, 291.

[22] van Amerongen, S., van Paradijs, J.: $A \& \mathcal{E} A$, 1989, 219, 195.

[23] Watson, M. G., King, A. R., Osborne, J.: $M N$ $R A S$, 1985, 212, 917.

[24] Winkler, C., Courvoisier, T. J.-L., Di Cocco, G., et al.: $A \& \&$ \& 2003, 411, L1.

[25] Galis, R. Proceedings of \%. INTEGRAL Workshop, Proceedings of Science, 2008.
[26] Hudec, R. et al.: Nuclear Physics B Proceedings Supplements, 2007, Vol. 166, p. 255-257.

[27] Pian, E. et al.: Observations of Blazars in Outburst with the Integral Satellite, in Triggering Relativistic Jets (Eds. William H. Lee, Enrico Ramírez-Ruiz) Revista Mexicana de Astronoma y Astrofisica (Serie de Conferencias) Vol. 27, 2007, Contents of Supplementary CD, p. 204.

René Hudec

E-mail: rene.hudec@gmail.com

Astronomical Institute

Academy of Sciences of the Czech Republic CZ-25165 Ondřejov, Czech Republic

Faculty of Electrical Engineering

Czech Technical University in Prague

Technická 2, CZ-16627 Prague, Czech Republic

Martin Blažek

Astronomical Institute

Academy of Sciences of the Czech Republic

CZ-25165 Ondřejov, Czech Republic

Faculty of Electrical Engineering

Czech Technical University in Prague

Technická 2, CZ-16627 Prague, Czech Republic

Věra Hudcová

Astronomical Institute

Academy of Sciences of the Czech Republic

CZ-25165 Ondřejov, Czech Republic 